\title{
Stellar model atmospheres with emphasis on velocity dynamics
}

\author{
Ch. Stütz ${ }^{1}$ and F. Kupka ${ }^{2}$ \\ ${ }^{1}$ Institut für Astronomie der Universität Wien Türkenschanzstraße 17, 1180 Wien, Austria \\ email: stuetz@jan.astro.univie.ac.at \\ ${ }^{2}$ Max-Planck-Institute for Astrophysics, Karl-Schwarzschild Str.1, 85741 Garching, Germany \\ email: fk@mpa-garching.mpg.de
}

\begin{abstract}
Recently a formalism for solving the open set of non-local hydrodynamic moment equations has been applied to the envelopes of A-stars by Kupka \& Montgomery (2002). Tests on numerical simulations and selected properties derived from stellar spectra such as average surface velocities and velocity skewness have shown the applicability of this RSM approach. In addition, a variety of improvements over existing modeling standards have been developed within and around the AMS group at the Institute for Astronomy in Vienna during the last couple of years. For example, individual elemental abundance patterns (Piskunov \& Kupka 2001) or stratification as an observed parameter (Shuliak et al. 2004) can be taken into account in our model atmospheres. Once the underlying programs, which currently operate as stand alone applications, are combined with a model atmosphere code, we will obtain a powerful and efficient tool that will allow us the investigation of a number of open problems in the physics of A-stars, such as more realistic models of the temperature gradients of cool CP2 stars.
\end{abstract}

Keywords. Convection, stars: atmospheres, stars: chemically peculiar

\section{Convection model}

An alternative to numerical simulations of convection, when the latter are unaffordable, are turbulence models based on the open set of non-local, hydrodynamic moment equations, the Reynold Stress models (RSM). Recently, Kupka \& Montgomery (2002) extended the Canuto \& Dubovikov (1998) RSM to stellar like scenarios including realistic microphysics, radiative losses in optically thin regions, and compressibility, for regimes of weak convection. A first application using realistic models of A-type main sequence stars has already shown that

- the mean velocities in the photosphere are comparable with typical values for macroand microturbulence parameters in these stars;

- He II and H I convection zones are thermally separated but dynamically connected and there is also considerable overshooting below the He II convection zone; and

- comparisons with mixing-length theory (MLT) convective fluxes and velocities immediately show the shortcomings of such local models (too small extension of mixed layers, uncertain calibration of mixing length).

\section{Atmospheric models}

In regimes where convection is weak, local FST (full spectrum turbulence) convection models such as the one by Canuto, Goldman and Mazzitelli (1996) have proven their usefulness in abundance analyses, pulsation research and investigations of magnetic fields, 


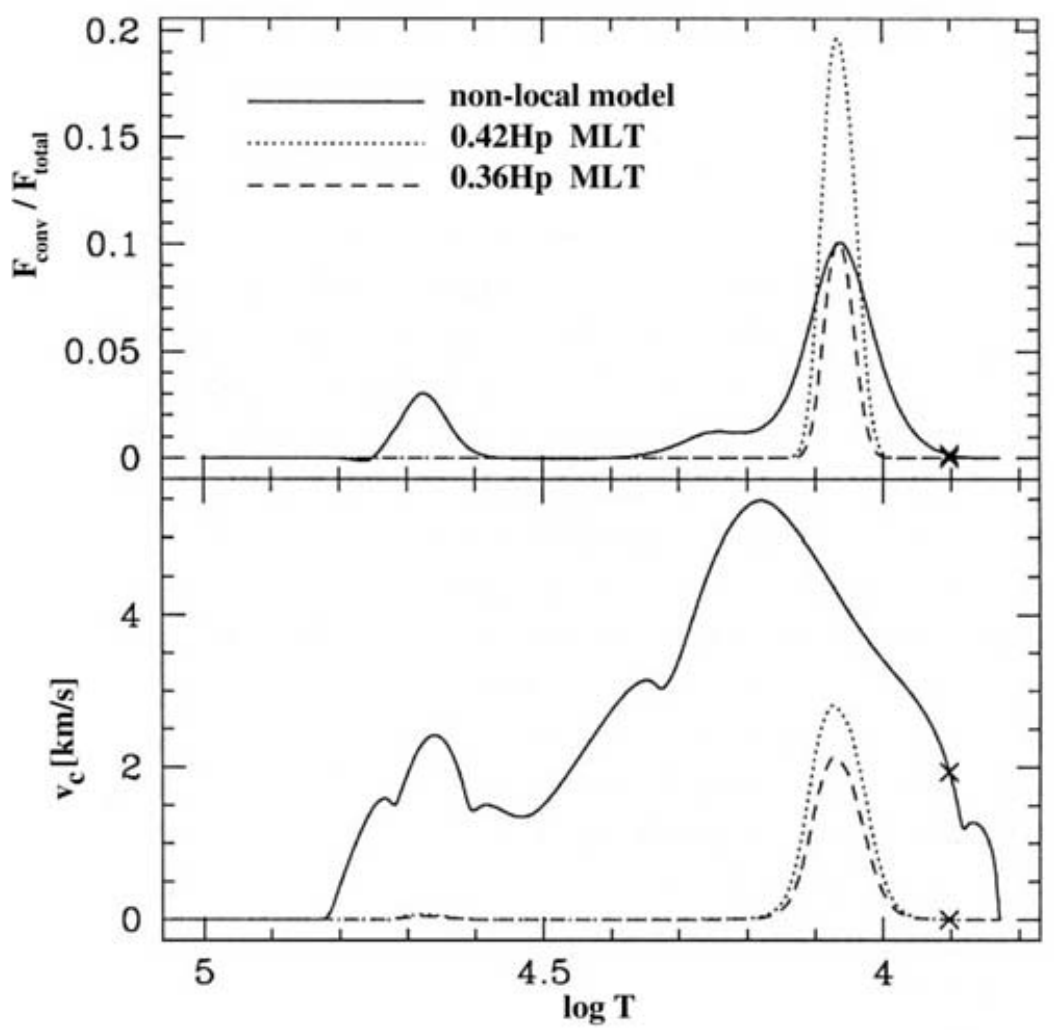

Figure 1. A comparison of convective fluxes and velocities for the non-local RSM and two MLT models $\left(\alpha=0.36\right.$ and $\left.0.42 H_{\mathrm{p}}\right)$, for $T_{\text {eff }}=8000 \mathrm{~K}$ and $\log g=4.4$. For a discussion of these calculations see Kupka \& Montgomery (2002).

but we have already found examples where we reach their limitations.

Opacity distribution functions are by far the fastest way to account for line opacities in atmosphere modeling. ODF precalculation for individual elemental abundance patterns (Piskunov \& Kupka 2001) considerably increases the usefulness of this approach.

Alternatively, including line opacities line-by-line during each iteration naturally supports individual element abundances, element stratification and depth dependent turbulent velocities. Shuliak et al. (2004) managed to develop such a line-by-line model atmosphere code which is fast enough for everyday use (1h total calculation time on a $1.8 \mathrm{GHz} \mathrm{PC})$.

We are currently working on the inclusion of molecular line absorbtion to extend our models to cooler regimes.

\section{Perspectives}

Some questions which we would like to address with these new generation of model atmospheres are: 
- Where is convection starting in the HR diagram and when is it becoming strong enough to compete against diffusion processes in stellar atmospheres?

- The hotter Böhm-Vitense gaps $((B-V)=0.2-0.3 \mathrm{mag})$ probably occur due to a sudden onset of strong convection in stellar atmospheres. The structure of these gaps depends on the metallicity and the age of the stellar sample. Do we have another way to independently derive these fundamental physical parameters by applying the new convection model?

- Can we explain and thus model line bisectors and convective line broadening consistently over a large parameter space?

- We might also be able to address questions such as overshooting, Li depletion or the transition from fast to slowly rotating Ap stars.

\section{Acknowledgements}

We would like to thank the IAU for supporting our participation at the IAU Symposium No. 224 through a travel grant. This research was supported by the Austrian Fonds zur Förderung der wissenschaftlichen Forschung (project P14984)

\section{References}

Canuto, V.M., Goldman, I., \& Mazzitelli, I., 1996, ApJ 473, 550

Canuto, V.M., Dubovikov, M.S., 1998, ApJ 493, 834

Kupka, F., Montgomery, M.H., 2002, MNRAS 330, L6

Piskunov, N., Kupka, F., 2001, ApJ 547, 1040

Shuliak, D., Tsymbal, V., Ryabchikova, T., Stütz, Ch., 2004, A\&\&A submitted 